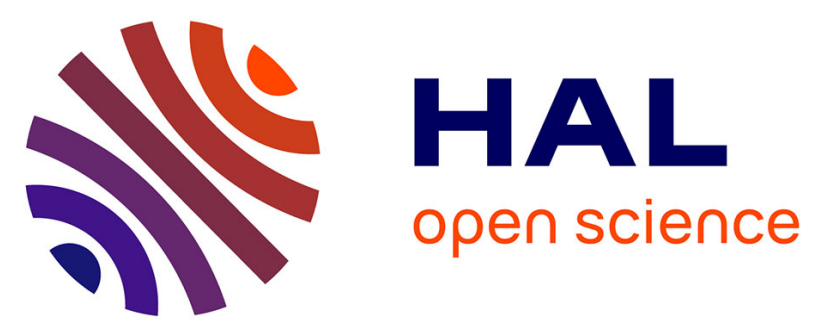

\title{
When giant vesicles mimic red blood cell's dynamics: swinging of two-phase vesicles in shear flow
}

\author{
Simon Tusch, Etienne Loiseau, Al-Hair Al-Halifa, Kamel Khelloufi, \\ Emmanuèle Helfer, Annie Viallat
}

\section{- To cite this version:}

Simon Tusch, Etienne Loiseau, Al-Hair Al-Halifa, Kamel Khelloufi, Emmanuèle Helfer, et al.. When giant vesicles mimic red blood cell's dynamics: swinging of two-phase vesicles in shear flow. Physical Review Fluids, 2018, 3 (12), 10.1103/PhysRevFluids.3.123605 . hal-01957925

\section{HAL Id: hal-01957925 \\ https://hal.science/hal-01957925}

Submitted on 17 Dec 2018

HAL is a multi-disciplinary open access archive for the deposit and dissemination of scientific research documents, whether they are published or not. The documents may come from teaching and research institutions in France or abroad, or from public or private research centers.
L'archive ouverte pluridisciplinaire HAL, est destinée au dépôt et à la diffusion de documents scientifiques de niveau recherche, publiés ou non, émanant des établissements d'enseignement et de recherche français ou étrangers, des laboratoires publics ou privés. 


\title{
When giant vesicles mimic red blood cell's dynamics: swinging of two-phase vesicles in shear flow
}

\author{
Simon Tusch ${ }^{1}$, Etienne Loiseau ${ }^{2}$, Al-Hair Al-Halifa ${ }^{1}$, Kamel Khelloufi ${ }^{2}$, Emmanuèle Helfer ${ }^{2}$,
} Annie Viallat ${ }^{2 *}$

1 Aix Marseille Univ, Marseille France

2 Aix Marseille Univ, CNRS, CINAM Marseille France

* annie.viallat@univ-amu.fr

\begin{abstract}
Red blood cells under shear flow present a specific swinging motion superimposed to a fluidlike tanktreading motion. Swinging is hypothesized to originate from periodic storage of shear energy in the cell membrane. Here we designed giant unilamellar vesicles with two lipid phases separated by a contact line, which swing and tanktread like red cells. We propose a model that quantitatively fits our data, finds the value of the contact-line tension and shows that swinging is due to the storage of elastic energy associated with the periodic modulation of the contact-line length during tanktreading.
\end{abstract}

\section{INTRODUCTION}

The dynamics of deformable microcapsules, such as giant unilamellar lipid vesicles $\left(G_{U V s}\right)^{1,2}$, polymer microcapsules ${ }^{3}$, elastic drops ${ }^{4}$ and red blood cells ${ }^{5}$ (RBCs) in suspension under shear flow is extensively studied because of its importance in proper blood circulation and drug delivery, the archetypal example being the RBC motion under shear flow. The coupling between the capsule elasticity and the suspending fluid, which depends on the constitutive mechanical properties displayed by microcapsules, is non-trivial and raises complex questions of non-linear physics and fluid structure interaction. The resulting richness of regimes of motion of these capsules is still not understood.

Prolate or oblate ellipsoidal capsules have two motions under shear flow known since the seventies, namely tumbling and tanktreading ${ }^{6,7,8}$. They are observed when the capsule is suspended in a fluid of respectively low and high viscosity compared to that of the internal fluid. Tumbling is analogous to a rigid-ellipsoid motion with a non-stationary orientation in flow (Fig. 1a). Note that GUVs, because of their high membrane flexibility, present also a tumbling motion with strong time-dependent shape deformations, called trembling ${ }^{9}$. Tanktreading is a droplet-like motion, characterized by the rotation of the shell and the internal fluid of the capsule around its center of mass, while the capsule maintains a stationary 
orientation with respect to the flow direction (Fig. 1c). In the following, we call 'membrane' the capsule shell in reference to GUVs and RBCs. In 1982, Keller and Skalak ${ }^{10}$ modeled the motion under shear flow of a fluid ellipsoid with a prescribed shape. They predicted both tumbling and tanktreading, and a 'tumbling to tanktreading' transition (T/TT transition) governed by two parameters, the viscosity contrast between the suspending fluid and the capsule inner fluid, and the ellipsoid aspect ratio.

Various other regimes of motion were recently discovered, such as swinging ${ }^{5}$, superimposed to tanktreading, which has been reported for both RBCs, protein-coated drops and polymer capsules, and rolling ${ }^{11}$ (Fig. 1b), kayaking ${ }^{12}$, flip-flopping ${ }^{13}$ and chaotic motion (in oscillatory shear flow) ${ }^{14}$ which have been observed for RBCs. These new regimes of motion were explained by accounting for the shear elasticity of the membrane, in addition to the viscosity contrast and the aspect ratio. The specific role of shear elasticity is best illustrated by the example of swinging. In this regime, the inclination of the tanktreading capsule oscillates about a mean angle. The strain of a local membrane element on a capsule deformed under shear flow periodically varies as the element moves along the capsule during tanktreading and therefore, so does the capsule shear-elastic energy (Fig. 1d). Moreover, when the stress-free shape of the capsule (i.e. the three-dimensional geometry of the membrane in which there is no shear stress ${ }^{15}$ ) is not spherical the membrane elements are not equivalent and the total shear strain and elastic energy ${ }^{16}$ of the capsule membrane also periodically vary during tanktreading, as illustrated in Fig. 1d for a capsule with an ellipsoidal stress-free shape. The dynamics of the system then depends on two time-dependent variables, the orientation angle of the capsule with respect to the flow and the position of the membrane elements on the capsule surface. During tanktreading these two coupled variables synchronize, leading to the periodic variation of the orientation angle of the capsule observed in swinging ${ }^{5,17}$.

The key phenomenon generating a large variety of motions is therefore believed to be the capacity of a capsule to periodically store energy during its flow motion. In addition to very compelling $3 \mathrm{D}$ numerical simulations ${ }^{12,13,18}$, the validity of this hypothesis is based on the experimental observation that tanktreading capsules with a shear modulus swing, whereas purely viscous GUVs tanktread with a steady inclination ${ }^{1,19}$. Moreover, shear-energy storage also explains why the T/TT transition depends on the shear rate for RBCs and not for GUVs. But so far, apart from polymer microcapsules for which swinging was incidentally reported ${ }^{3}$, RBCs are the only capsules combining shear-elasticity and non-spherical stress-free shape for which swinging and shear-rate induced T/TT transition was extensively experimentally, theoretically, and numerically reported. 
Here, we consider GUVs whose membrane consists of two lipid phases separated by a contact line. These GUVs have prolate ellipsoidal shape under shear flow. When they tanktread the contact line rotates from the meridian zone of the GUV to its equatorial one. The length of the contact line therefore varies. As the energy of the contact line depends on its length, it is expected to periodically vary during tanktreading. This system is therefore a good candidate to exhibit swinging under shear flow and shear-rate induced T/TT transition. The aim of this work is to comfort the causality between storage energy and swinging by combining an experimental and theoretical work on designed-on-purpose two-phase GUVs, able to periodic store and tune their contact-line energy during the flow motion.

\section{MATERIALS AND METHODS}

\section{A. GUV preparation}

Two-phase vesicles were prepared using the electroformation method at $60^{\circ} \mathrm{C}^{20}$ from a mixture of dioleoyl-phosphatidylcholine (DOPC), dipalmitoyl-phosphatidylcholine (DPPC) and cholesterol (Chol) in mass ratio 4:4:2 at a final concentration of $2 \mathrm{mg} / \mathrm{mL}$ in methanol:chloroform 1:9. N-(lyssamine rhodamine B sulfonyl)-dipalmitoylphosphatidylethanolamine (Rh-DPPE) $(1 \mathrm{mg} / \mathrm{ml})$ (5\% in volume) was added to the mixture for fluorescence microscopy observation (all lipids from Avanti Polar Lipids). Vesicles were electroformed in $0.1 \mathrm{M}$ sucrose solution and subsequently diluted in 0.105 to $0.2 \mathrm{M}$ glucose solution to control vesicle deflation. At room temperature the ternary lipid mixture spontaneously forms two stable liquid-ordered $\left(\mathrm{L}_{\mathrm{o}}\right)$ and liquid-disordered $\left(\mathrm{L}_{\mathrm{d}}\right)$ phases separated by a contact line. Rh-DPPE preferentially partitions in the $\mathrm{L}_{\mathrm{d}}$ phase.

For certain GUVs, the surface areas of the two liquid-ordered and liquid-disordered phases were equal $\left(50 \% \mathrm{~L}_{\mathrm{o}} / 50 \% \mathrm{~L}_{\mathrm{d}}\right)$. For several GUVs, some vesiculation probably occurred and the membrane surface area of the two lipid phases was different, up to $30 \% \mathrm{~L}_{\mathrm{o}} / 70 \% \mathrm{~L}_{\mathrm{d}}$.

Flow experiments: Vesicles were gently injected in a parallelepiped flow chamber with four optical faces $\left(1 \times 10 \times 45 \mathrm{~mm}^{3}\right)$, let sediment, and observed using a microscope (LEICA) tilted at $90^{\circ}$ with a $\times 20$ objective and a camera Andor Neo sCMOS at $20 \mathrm{fps}$, as previously described $^{1}$. The flow was applied using a syringe pump (ProSense B.V model NE-1000) at shear rates ranging from 1 to $12 \mathrm{~s}^{-1}$. We experimentally observed 10 different GUVs at various shear rates (from 3 to $11.5 \mathrm{~s}^{-1}$ ), among which 8 were in the tanktreading/swinging regime and whose motion was analyzed. Within this set of 8 vesicles, 4 were studied at varying shear rates ( 3 to 4 values). The geometric parameters (size, reduced volume and $\mathrm{L}_{\mathrm{o}} / \mathrm{L}_{\mathrm{d}}$ 
partitioning), the capillary number, and the shear rates used for the 10 vesicles are listed in Table 1.

\author{
B. Image analysis.
}

In-house Matlab routines were developed to track the vesicles and extract their geometrical parameters. The shape of the vesicle projection in the shear plane was fitted by an ellipse. The long and short axis of the ellipse were identified: we determined their respective lengths $\left(2 a_{1}\right.$ and $2 a_{2}$ ) and the orientation of the long axis with respect to the flow direction, defined as the inclination angle $\theta$. The projection of the phase separation line on the ellipse was identified: we derived the length $l$ of the contact line on the whole vesicle (considered as an ellipsoid of revolution around its long axis) and determined the phase angle $\alpha$. It is defined as the angle formed by the major axis of the ellipse and the bisector of the two segments each connecting the center of the ellipsoid to the contact line (figure $2 \mathrm{~b}$ and Supplemental Material ${ }^{21}$ ).

\title{
III. RESULTS AND DISCUSSION
}

\section{A. Observations}

A typical experiment is illustrated in Fig. 2. Without flow, GUVs rest on the bottom surface on the flow chamber and have a non-axisymmetric bilobed shape, each lobe consisting of $\mathrm{L}_{\mathrm{o}}$ and $\mathrm{L}_{\mathrm{d}}$ domains, respectively, separated by a contact line (Fig. 2a). The equilibrium shape of GUVs minimizes the total energy of their membrane resulting from the sum of bending energy (Helfrich energy), buoyancy, and contact-line energy.

Under low shear rate $(\dot{\gamma})$, GUVs elongate and move on the surface along the flow direction. When $\dot{\gamma}$ increases, they unbind and stabilize their position at a given distance from the substrate, where the lift force balances the vesicle buoyancy ${ }^{1}$. The shape deformation of GUVs depends on their reduced volume $v$, a non-dimensional number defined as the ratio of the vesicle volume $\mathrm{V}$ to the volume of the sphere with the same surface area A: $v=$ $\frac{3 V}{4 \pi(A / 4 \pi)^{3 / 2}},(v \leq 1, v=1$ for a sphere). The lower the reduced volume the higher the shape deformation. GUVs with high reduced volume $(v \geq 0.9)$ display a quasi-stationary shape tending to a prolate ellipsoid and tanktread as reported for single-phase GUVs ${ }^{1}$ and $\mathrm{RBCs}^{8,5}$. The vesicle (GUV\#1 in Table 1) shown in Figure 2c and in ref (22) is a typical one with an average reduced volume $\langle v\rangle=0.96 \pm 0.04$. The rotation of the membrane at low $\dot{\gamma}\left(<10 \mathrm{~s}^{-1}\right)$ is in the shear plane with a stable orbit. Noteworthy, the contact line remains in a plane 
perpendicular to the shear plane. At higher $\dot{\gamma}$, the membrane movement is more complex with a transitory out-of-shear plane contribution as observed when increasing the shear rate to 11.5 $\mathrm{s}^{-1}$ (GUV \#1, Fig. 2d and ref (23)). At intermediate reduced volume, GUVs strongly deform during tanktreading as shown for GUV\#9 in Table $1: v \approx 0.8$ (Fig. 2e and ref (24)). When the contact line passes in the meridian zone, the GUV looks like two adhered vesicles, and the contact line is shorter than for ellipsoidal GUVs. At lower reduced volume, GUVs tumble (see Fig. 2f and see Supplemental Material ${ }^{25}$, GUV \#10 in Table 1: $v \approx 0.7$ ), thus avoiding the strong increase in the contact line that would be required when passing in the meridian zone of an elongated shape. Tumbling of GUVs whose internal fluid and suspending fluid have the same viscosity has never been previously experimentally observed.

\section{B. Motion and shape analysis}

The motion is characterized by four geometrical parameters (Fig. 2b), the inclination angle $\theta$ between the GUV major axis and the flow direction, the phase angle $\alpha$ between the major axis and the bisector of the two segments each connecting the center of the ellipsoid to the contact line, the length of the contact line $l$ between the two lipid phases, and the GUV flattening defined as $f=\left(a_{1}-a_{2}\right) / a_{1}$, where $a_{1}$ and $a_{2}$ are the half-lengths of the long and short axis, respectively. Temporal evolutions of $\theta, \alpha, l$ and $f$ are shown in Fig. 3a for a quasispheroid GUV (GUV \#6 in Table 1). Here the GUV flows very close to the surface and an asymmetry in $\theta$-temporal evolution is observed. The origin of this asymmetry is not clear. We hypothesize that it results from the dissymmetry between the Lo/Ld partitioning on the GUV combined with its very close vicinity to the wall. Indeed, this GUV being almost spherical experiences only a small unbinding force. Typically, GUVs swing ( $\theta$ angle) and their swinging period is half that of tanktreading ( $\alpha$ angle), similarly to observations on $\mathrm{RBCs}^{5}$. A significant periodic flattening (typically 20\%) and contact-line length variation are observed at the swinging period. Another vesicle (GUV \#3 in Table 1) is shown, see Supplemental Material ${ }^{26}$. The $\dot{\gamma}$-effect on swinging and tanktreading is displayed in Fig. 3b-c by showing the evolution of $\theta$ and $\alpha$ versus the adimensioned time $\dot{\gamma} t$. Supplemental $\mathrm{Material}^{27}$ shows $\alpha$-dependence on real time, superimposed with the model fits (see below). The amplitude of the oscillations on the $\theta$-curves and the tanktreading period very significantly decrease with increasing shear rate. The phase relationships between $f, \theta$ and $l$ are shown in Fig. 4a. $f$ and $l$ have a $180^{\circ}$ phase shift: the maximal contact line, observed when it lies in the plane containing the long GUV axis, is reached for the minimal GUV elongation, 
thus contributing to limit the contact-line length and its associated elastic energy. $l$ and $\theta$ have a $90^{\circ}$ phase shift. The $\dot{\gamma}$-dependence of the tanktreading period and of the swinging amplitude for all analyzed GUVs (GUVs \#1-8 in Table 1) are displayed in Fig. 4b. Both parameters vary linearly in $\dot{\gamma}^{-1}$. All tanktreading periods fall on the same curve whereas the swinging amplitudes, more sensitive to the reduced volume and the partitioning between the lipid phases fall in the narrow shade region.

\section{Model}

We rely on the analytical model of Keller and Skalak (KS) and on its extension for RBCs ${ }^{5}$ to include in the KS model the energy periodically stored by the contact line of the GUV membrane. The KS model treats a capsule as a fluid ellipsoid of revolution delimited by a membrane enclosing a viscous liquid. The membrane elements are prescribed to rotate along elliptical trajectories parallel to the shear plane. KS established the equation of evolution of $\theta$ by stating that at equilibrium the total moment exerted by the external fluid on the capsule vanishes. In addition, the movement satisfies the conservation of energy, i.e. the rate of dissipation of energy in the capsule must equal the rate at which work is done by the external fluid on the capsule. KS calculated both rates assuming viscous energy dissipation in the capsule. In the case of RBCs, the AFV-SS ${ }^{5}$ model added to this latter contribution that of the elastic power stored in the periodic local shear deformations of the membrane during tanktreading. Two coupled differential equations for $\mathrm{d} \theta / \mathrm{d} t$ and $\mathrm{d} \alpha / \mathrm{d} t$ were then obtained, which allowed to numerically calculate the capsule motion.

Here we adapt this model to GUVs, taken as prolate ellipsoids of revolution (identical short axes $a_{2}$ and $a_{3}$ ) and divided in two phases and we introduce the contribution of the contactline energy. For the sake of simplicity, we describe the calculation when the vesicle displays a membrane of equal surface area between the two phases $\left(50 \% / 50 \% \mathrm{~L}_{\mathrm{o}} / \mathrm{L}_{\mathrm{d}}\right)$. The extension of the model for surface areas of different ratios between the two phases are described in Supplemental Material ${ }^{28}$. We write the balance of energy as $W p=D v+P_{L}$, where $D v$ is the rate at which the work $W p$ is transferred by the external fluid on the GUV and $P_{L}$ is the time derivative of the line energy. $P_{L}=\mathrm{d} E_{L} / \mathrm{d} t$, where $E_{L}=\lambda_{L} \cdot l(\alpha)$ is the line energy, $\lambda_{L}$ is the line tension, $l(\alpha)$ is the length of the elliptical separation line when the phase angle is equal to $\alpha$. The value $l(\alpha)$ is derived from the Ramanujan approximation for the circumference of an ellipse :

$$
l(\alpha)=\pi\left(3\left(a_{l}(\alpha)+a_{3}\right)-\sqrt{\left(3 a_{l}(\alpha)+a_{3}\right)\left(a_{l}(\alpha)+3 a_{3}\right)}\right)
$$


with

$$
a_{l}(\alpha)=\sqrt{a_{1}^{2}(\sin \alpha)^{2}+a_{2}^{2}(\cos \alpha)^{2}}
$$

The $\alpha$-derivative of $l(\alpha)$ is

$$
l^{\prime}(\alpha)=\pi a_{l}^{\prime}(\alpha)\left(3-\frac{3 a_{l}(\alpha)+5 a_{3}}{\sqrt{\left(3 a_{l}(\alpha)+a_{3}\right)\left(a_{l}(\alpha)+3 a_{3}\right)}}\right)
$$

with

$$
a_{l}^{\prime}(\alpha)=\frac{\left(a_{1}^{2}-a_{2}^{2}\right) \sin 2 \alpha}{2 a_{l}(\alpha)}
$$

The value of $P_{L}$ therefore writes as

$$
P_{L}=\frac{d \alpha}{d t} \lambda_{L} l^{\prime}(\alpha)
$$

Conservation of energy yields a second differential equation in complement to that derived by KS from the equilibrium of moments.

The two adimensioned coupled equations are

$$
\begin{aligned}
& \frac{d \theta}{d t}=\dot{\gamma}\left(-\frac{1}{2}+c_{11} \cos 2 \theta+c_{12} F(\alpha)\right) \\
& \frac{d \alpha}{d t}=\dot{\gamma}\left(-c_{21} \cos 2 \theta-c_{22} F(\alpha)\right)
\end{aligned}
$$

where $F(\alpha)$ is the adimensionned ratio $\frac{l^{\prime}(\alpha)}{l_{\max }^{\prime}}$ with $l_{\max }^{\prime}$ being the maximal value of $l^{\prime}(\alpha)$ and $V$ the vesicle volume, $c_{21}=\frac{\eta_{e} f_{3}}{\eta_{e} f_{2}-\eta_{i} f_{1}}, c_{22}=-\frac{\lambda_{L} l_{\text {max }}^{\prime}}{\dot{\gamma}\left(\eta_{e} f_{2}-\eta_{i} f_{1}\right) V}, c_{11}=\frac{1}{2} \frac{a_{1}^{2}-a_{2}^{2}}{a_{1}^{2}+a_{2}^{2}}+\frac{2 a_{1} a_{2}}{a_{1}^{2}+a_{2}^{2}} c_{21}$, $c_{12}=\frac{2 a_{1} a_{2}}{a_{1}^{2}+a_{2}^{2}} c_{22}, \eta_{e}$ and $\eta_{i}$ are the viscosities of the suspending fluid and of the inner fluid, respectively, and $f_{1}, f_{2}$ and $f_{3}$ are the geometrical factors defined by KS.

When the viscosity of the external and the inner fluid are equal, $c_{21}$ and $c_{11}$ are only geometrical factors and the ratio $\frac{c_{22}}{c_{21}}=\frac{\lambda_{L} l_{\max }^{\prime}}{\dot{\gamma} \eta_{e} f_{3} V}$ represents the ratio of the maximal line energy to the fluid energy. Therefore, it clearly appears from (7) that $\frac{c_{22}}{c_{21}}$ controls the nature of the motion. At low values the GUV tanktreads and swings whereas above a critical value it tumbles. For a given GUV geometry, the regime of motion is determined by the ratio $\frac{\lambda_{L}}{\dot{\gamma} \eta_{e}}$. The $\dot{\gamma}$-behavior of $\theta$ and $\alpha$ in both tanktreading and tumbling regimes are shown in Fig. 5 . The phase trajectories of $\theta$ versus $\alpha$ are illustrated by their limit cycle in Fig. 6 for a GUV with $40 \%$ of the surface area covered by the $\mathrm{L}_{\mathrm{o}}$ phase. Corresponding Movies in 
Supplemental Material ${ }^{29,30,31}$ display the evolution of $\theta$ vs $\alpha$ that led to the curves in Fig. 6. Interestingly, at the tumbling-tanktreading transition, an intermittent regime is observed, similar to that reported at the tumbling-tanktreading transition of $\mathrm{RBCs}^{5,15,16}$.

Finally, we numerically solved the equations to fit the motions of GUV \#1 and GUV \#7 in Table 1 under the 4 different shear rates experimentally studied. We used the average values of $a_{1}$ and $a_{2}$ measured on each GUV, and set $a_{2}=a_{3}$; the viscosities of the dilute sucrose and glucose solutions, $\eta_{i}$ and $\eta_{e}$, were taken identical and equal to $1.2 \cdot 10^{-3}$ Pa.s. All these parameters were determined with an uncertainty of approximately $10 \%$. The line tension $\lambda_{L}$ was the only adjustable parameter. It was set to $15 \mathrm{pN}$ to correctly fit the 8 experiments (4 shear rates, two vesicles). It is in good agreement with experimental data reported in the literature for DOPC/DPPC/Chol $\left(\approx 3 \mathrm{pN}\right.$ for $\left.20 \% \mathrm{Chol}^{32}\right)$ and with modeling ${ }^{33}(4-35 \mathrm{pN})$. Model predictions and experiments are in good quantitative agreement concerning the $\dot{\gamma}$ dependence of the swinging period and inclination angle (Figs. 3-4 and Supplemental Material $^{27}$ ), for GUVs \#1 and \#7, and Supplemental Material ${ }^{26}$ for the GUV\#3). Furthermore, the model fits the tumbling motion of GUV \#10 (Fig. 2f) under a shear rate of $3 \mathrm{~s}^{-1}$. By using a line tension of $100 \mathrm{pN}$ (6.5 higher than for other vesicles), the model correctly predicts the observed tumbling period (5s) and the quasi-null oscillation of the angle $\alpha$ (see Supplemental Material $^{34}$ ). The neck shape of this GUV at the contact line indeed suggests a high value of the line tension.

A question also arises about the potential effect on swinging of the difference in bending energies between the two lipid phases ${ }^{35}$. For instance, 2D numerical simulations performed on two-component 2D-vesicles with a difference in bending energy between the lipid phases predicted a T/TT transition triggered by an increase in shear rate ${ }^{36,37}$. In this model, however, the contact line being reduced to two points cannot drive an energy variation. As noticed in ref. 22, values of bending and line energy do not lie in the same range. Briefly, the variation of bending energy during tanktreading is of the order of $\pi \frac{\Delta \kappa}{\kappa} \kappa \approx 0.2 \pi 10^{-19} \mathrm{~J}$, where $\kappa \approx 10^{-19} \mathrm{~J}$ is the bending energy of the lipid membrane ${ }^{38}$ and $\frac{\Delta \kappa}{\kappa}$, the variation ratio between the two lipid phase bending energy, is of the order of $0.2^{22}$. The variation of line energy is $2 \pi \frac{\Delta R}{R} R \sigma \approx 2 \pi 0.210^{-16} \mathrm{~J}$, where the contact line radius $R$ is of the order of $10^{-5} \mu \mathrm{m}$, the variation rate of $\mathrm{R}$ during tanktreading, $\frac{\Delta R}{R} \approx 0.2$, and the line tension $\sigma \approx 10^{-11} \mathrm{~N}$. The line energy is therefore three orders of magnitude larger than the bending energy, thus showing that this latter parameter does not drive the observed GUV regimes of motion. It should 
however be noted that for GUVs with an equally distributed membrane surface area between the two phases, the bending energy does not change during tanktreading. Indeed, these GUVs present a central symmetry. For instance, when the DPPC-rich zone rotates, the membrane element at the front of this zone reaches a region on the GUV shape where the curvature is the same as that the membrane element at the rear of this zone just left. The bending energy is however important for the local continuous membrane geometry at the contact line. The difference in orders of magnitude between bending and line energy clearly appears in ref. 24, where tumbling is observed on extremely deflated deformable 2D-GUVs and the swinging is motion is mixed with shape variations, which is not experimentally observed in line-energy controlled experimental GUV motion.

\section{CONCLUSION}

In conclusion we show that the two-phase GUVs tumble, swing and tanktread like RBCs due to their non-symmetric rest shape and ability to periodically store elastic energy in their contact line. By quantitatively fitting experimental data, our model supports the interpretation of RBC swinging motion, based on periodic elastic energy storage in the cell membrane with an asymmetric stress-free shape.

Acknowledgments: We thank LAI (Aix Marseille Univ, CNRS, Inserm) for hosting S.M. and A.-H. A. - H. during several months. 
Table 1. Shape parameters of the 10 vesicles from the study, and the shear rates under which they were observed.

\begin{tabular}{|c|c|c|c|c|c|c|c|}
\hline $\begin{array}{c}\text { GUV } \\
\#\end{array}$ & $\begin{array}{c}\text { Mean } \\
\text { radius } \\
\langle\boldsymbol{r}> \\
(\boldsymbol{\mu m})\end{array}$ & $\begin{array}{c}\text { Flattening } \\
\boldsymbol{f}\end{array}$ & $\begin{array}{c}\text { Reduced } \\
\text { volume } \\
\boldsymbol{v}\end{array}$ & $\begin{array}{c}\mathbf{L}_{\mathbf{o}} / \mathbf{L}_{\mathbf{d}} \\
\mathbf{p a r t i t i o n i n g} \\
(\boldsymbol{\%} / \boldsymbol{\%})\end{array}$ & $\begin{array}{c}\text { Capillary number } \\
\eta_{e} r^{3} \dot{\gamma}\end{array}$ & $\begin{array}{c}\text { Shear rate } \\
\boldsymbol{( \mathbf { s } ^ { - 1 } )}\end{array}$ & $\begin{array}{c}\text { Regime } \\
\text { of } \\
\text { motion }\end{array}$ \\
\hline 1 & 15.6 & 0.35 & 0.96 & $32 / 68$ & $148 / 243 / 342 / 437$ & $3.9 / 6.4 / 9 / 11.5$ & TT \\
\hline 2 & 17.4 & 0.52 & 0.88 & $49 / 51$ & $205 / 327 / 406$ & $3.9 / 6.2 / 7.7$ & TT \\
\hline 3 & 17 & 0.23 & 0.98 & $46 / 54$ & 192 & 3.9 & TT \\
\hline 4 & 19.6 & 0.32 & 0.96 & $37 / 63$ & 294 & 3.9 & TT \\
\hline 5 & 21 & 0.48 & 0.91 & $52 / 48$ & 565 & 6.1 & TT \\
\hline 6 & 15.8 & 0.24 & 0.98 & $28 / 72$ & 118 & 3 & TT \\
\hline 7 & 22 & 0.30 & 0.97 & $47 / 53$ & $415 / 681 / 958 / 1224$ & $3.9 / 6.4 / 9 / 11.5$ & TT \\
\hline 8 & 24 & 0.34 & 0.96 & $43 / 57$ & $539 / 884 / 1244 / 1590$ & $3.9 / 6.4 / 9 / 11.5$ & TT \\
\hline 9 & 21 & 0.62 & 0.82 & $38 / 62$ & 361 & 3.9 & TT \\
\hline 10 & NA & NA & $<0.71$ & $53 / 47$ & & 3 & T \\
\hline
\end{tabular}

The mean radius $\langle r\rangle$ is the average value of long and short axis lengths $\left(2 a_{1}\right.$ and $2 a_{2}$, respectively) of the ellipse fitting the vesicle projection.

The flattening $f$ characterizes the shape aspect ratio at maximal GUV elongation.

The reduced volume $v$ is the ratio of the vesicle volume to the volume of a sphere with the same surface area. GUVs \#1-9 were approximated by an ellipsoid of revolution, to calculate their volume and surface area. In the peculiar case of GUV \#10 which looks like two adhered spheres (one in $\mathrm{L}_{\mathrm{o}}$ phase, the other one in $\mathrm{L}_{\mathrm{d}}$ phase), the vesicle could not be fitted by an ellipsoid: the volume and surface area were calculated by considering the sum of the 2 spheres, leading to an overestimation of the reduced volume.

The $\mathrm{L}_{\mathrm{o}} / \mathrm{L}_{\mathrm{d}}$ partitioning is the surface area percentages of the $\mathrm{L}_{\mathrm{o}}$ (non-fluorescent) and $\mathrm{L}_{\mathrm{d}}$ (fluorescent) phases.

The capillary number was calculated by using $\eta_{e}=10^{-3} \mathrm{~Pa}$, the bending energy $\kappa=$ $10^{-19} \mathrm{~J}, r$ is the mean radius and $\dot{\gamma}$ is the shear rate.

The regime of motion of the vesicles is either tanktreading/swinging (TT) or tumbling (T). 


\section{Figure captions}

Figure 1. a-c) Schematics of RBCs successively undergoing tumbling, rolling and tanktreading at increasing shear rate. The rolling regime is specific to RBCs and does not occur with vesicles. d) During swinging the elastic energy stored by membrane elements moving along the capsule contour varies periodically.

Figure 2. a) Vesicle at rest on the flow chamber surface. b) Parameters measured to characterize vesicle motion: vesicle size and shape $\left(a_{1}, a_{2}, a_{3}\right)$, orientation to flow $(\theta)$, phase angle $(\alpha)$, contact line length $(l)$. c) Typical vesicle of high reduced volume (mean radius $\langle r\rangle$ $=15.6 \mu \mathrm{m}$, mean flattening $\langle f\rangle=0.3$, reduced volume $v \approx 0.95$ ) exhibiting tanktreading/swinging motion under a shear rate of $3.9 \mathrm{~s}^{-1}$, with a mean inclination angle $\langle\theta\rangle$ $\approx 30^{\circ}$ (GUV \#1 in Table 1). d) Same vesicle as in c) undergoing complex motion with out-ofplane orientational changes of the membrane under a higher shear rate of $11.5 \mathrm{~s}^{-1}$. The lipid phases change hemispheres during tanktreading. e) A deflated vesicle with an intermediate reduced volume $(v \approx 0.8)$ strongly deforms during tanktreading under a shear rate of $3.9 \mathrm{~s}^{-1}$ (GUV \#9 in Table 1). When the contact line is in the meridian zone the vesicle is no longer ellipsoidal but resembles two vesicles adhered together, each consisting of a lipid phase. When the contact line is in the equatorial zone the vesicle has an ellipsoidal shape. f) A highly deflated vesicle with a low reduced volume $(v \approx 0.7)$ tumbling under a shear rate of $3.9 \mathrm{~s}^{-1}$ (GUV \#10 in Table 1). Scale bars: $20 \mu \mathrm{m}$.

Figure 3. a) Temporal evolution of $\theta, \alpha, f$ and $l$ of a typical tranktreading/swinging vesicle (mean radius: $\langle r\rangle=15.8 \mu \mathrm{m},\langle f\rangle \approx 0.2$ ) under a shear rate of $3 \mathrm{~s}^{-1}$ (GUV \#6 in Table 1). b-c) Phase $(\alpha)$ and inclination $(\theta)$ angles as function of the adimensioned time for the vesicle shown in Fig. 2c, at three shear values (GUV \#1 in Table 1). The solid lines are fits from Eqs. (6-7) using the vesicle shape parameters $\left(a_{1}=19.2 \mu \mathrm{m}, \mathrm{a}_{2}=\mathrm{a}_{3}=12 \mu \mathrm{m}\right)$ and a line tension of 15 $\mathrm{pN}$.

Figure 4. a) $l$-dependence of the flattening $f$ and the inclination angle $\theta$ for the vesicle shown in Fig. 3a (GUV \#6 in Table 1). Insets show the vesicle configurations at extremal $l$-values. b) Shear-rate dependence of the tanktreading period $T$ and of the swinging amplitude $\Delta \theta$ in LogLog scale, for all the analyzed vesicles. Diamond symbols correspond to the 4 vesicles studied under several shear rates (one color per vesicle) whereas all other symbols correspond to vesicles observed under a single shear rate (see Table 1 for details). The thick solid lines are specific fits for two vesicles (GUV \#1, purple diamonds and GUV \#7, blue diamonds) calculated from the model using a line tension of $15 \mathrm{pN}$ and the vesicle respective shape parameters. Slope -1 expected in the low-line-tension limit is indicated. The shaded region on the $\Delta \theta$-plot is a guide to the eye: it contains the scattered data of all vesicles including the 2 fits at different shear rates with exhibit the same slope.

Figure 5. Theoretical phase (top) and inclination (bottom) angles versus time for 3 shear rates calculated from the model using a line tension of $150 \mathrm{pN}$ for a vesicle of flattening $f=0.5$ and reduced volume $v \approx 0.9$. Left: tumbling motion, $\alpha$ oscillates and $\theta$ rotates; center: swingingtanktreading motion near the transition, $\alpha$ rotates and $\theta$ oscillates in an asymmetric manner and with a large amplitude; right: swinging-tanktreading motion far from the transition, $\alpha$ rotates and $\theta$ oscillates with a small amplitude. Note the difference in the form of the $\alpha(t)$ curve near and close the tumbling/tanktreading transition. 
Figure 6. Theoretical phase trajectories of $\theta$ vs $\alpha$. Left: limit cycle for the tumbling motion (shear rate of $0.8 \mathrm{~s}^{-1}$ ), $\alpha$ oscillates and $\theta$ rotates; center: limit cycle of an intermittent regime at the tumbling-tanktreading transition (shear rate of $1.2 \mathrm{~s}^{-1}$ ); right: limit cycle for the swinging-tanktreading motion (shear rate of $6 \mathrm{~s}^{-1}$ ), $\alpha$ rotates and $\theta$ oscillates. The corresponding animations of the temporal evolutions of $\theta$ vs $\alpha$ are available in Supplemental Material29, 30 and 31. 
FIGURES

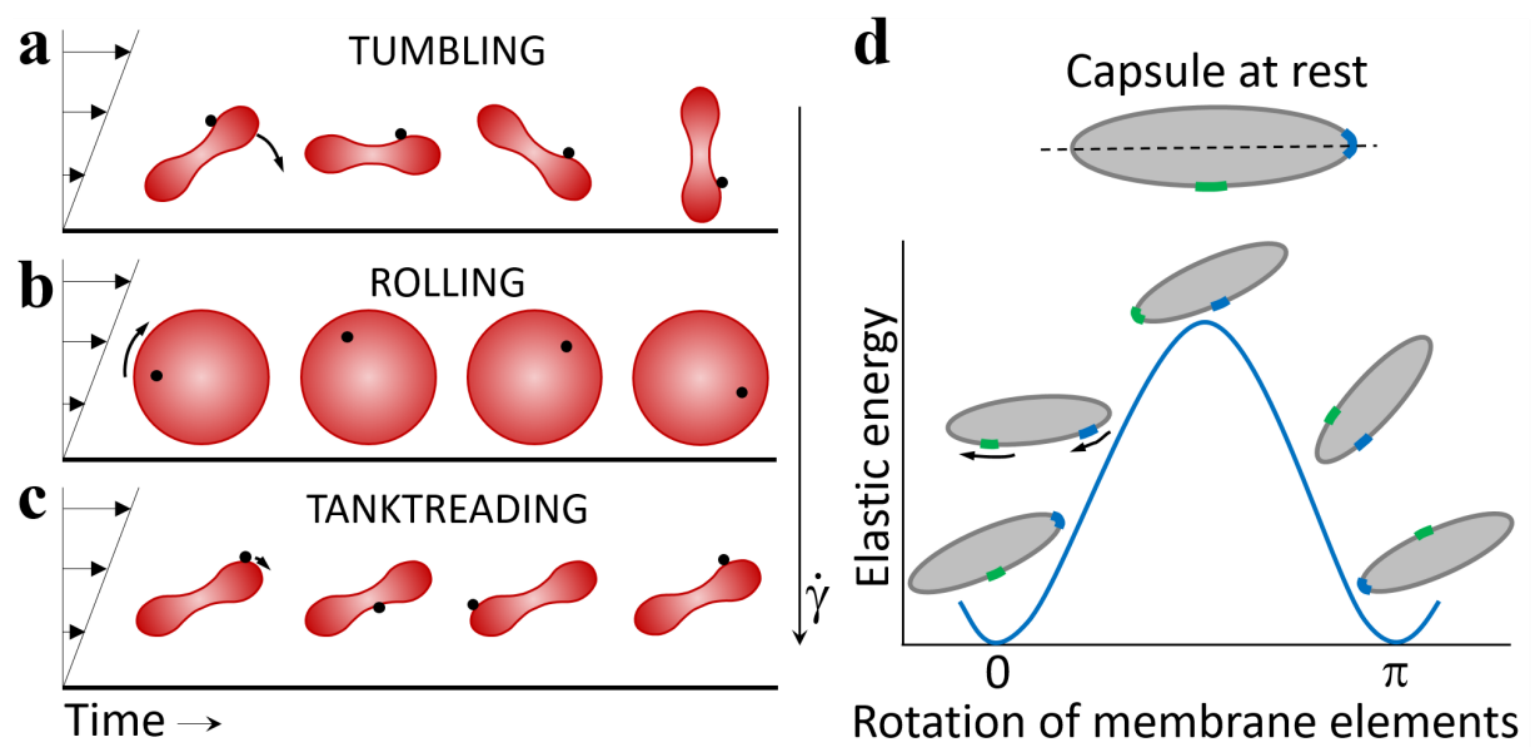

FIGURE 1 

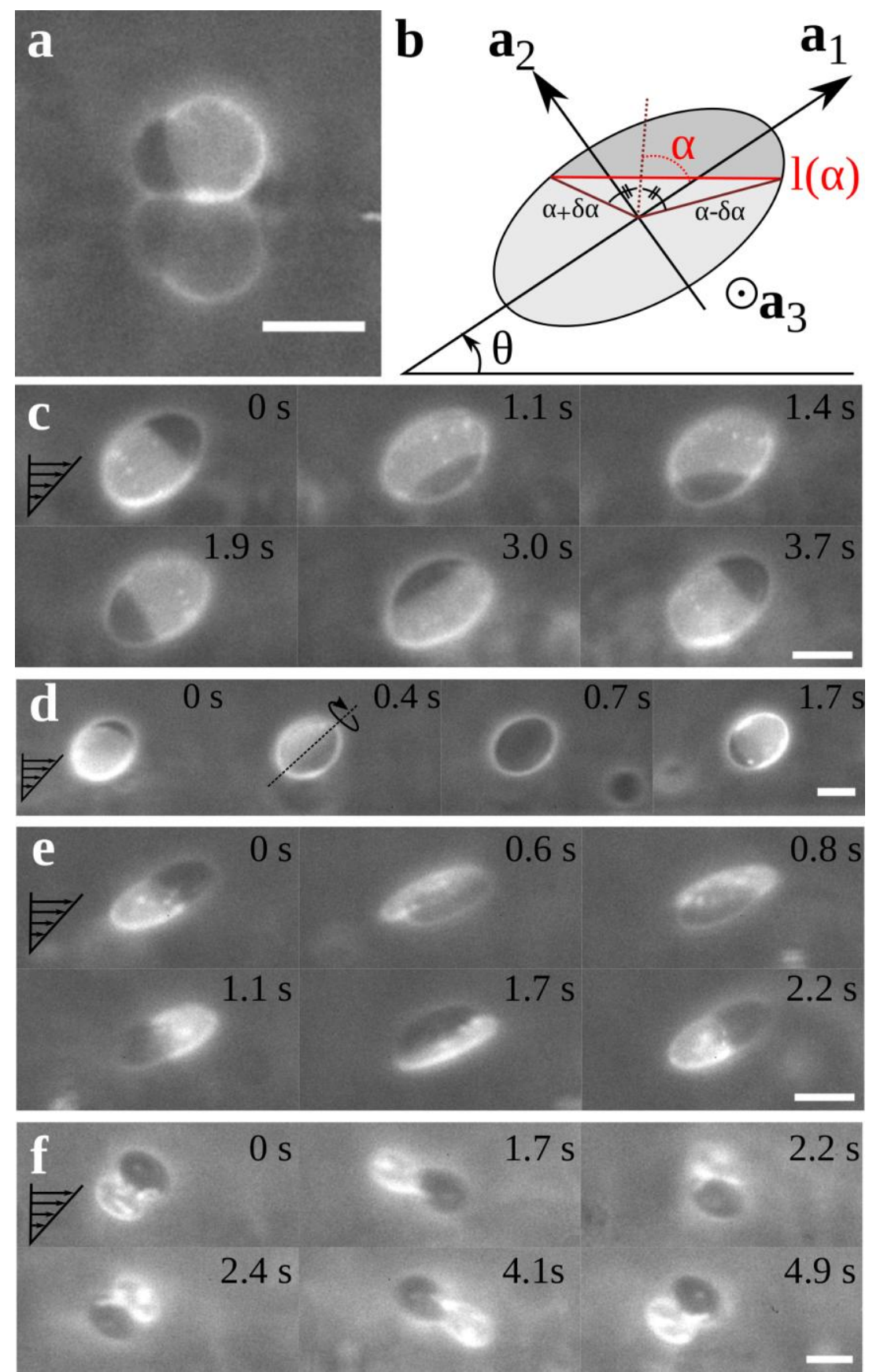

FIGURE 2 

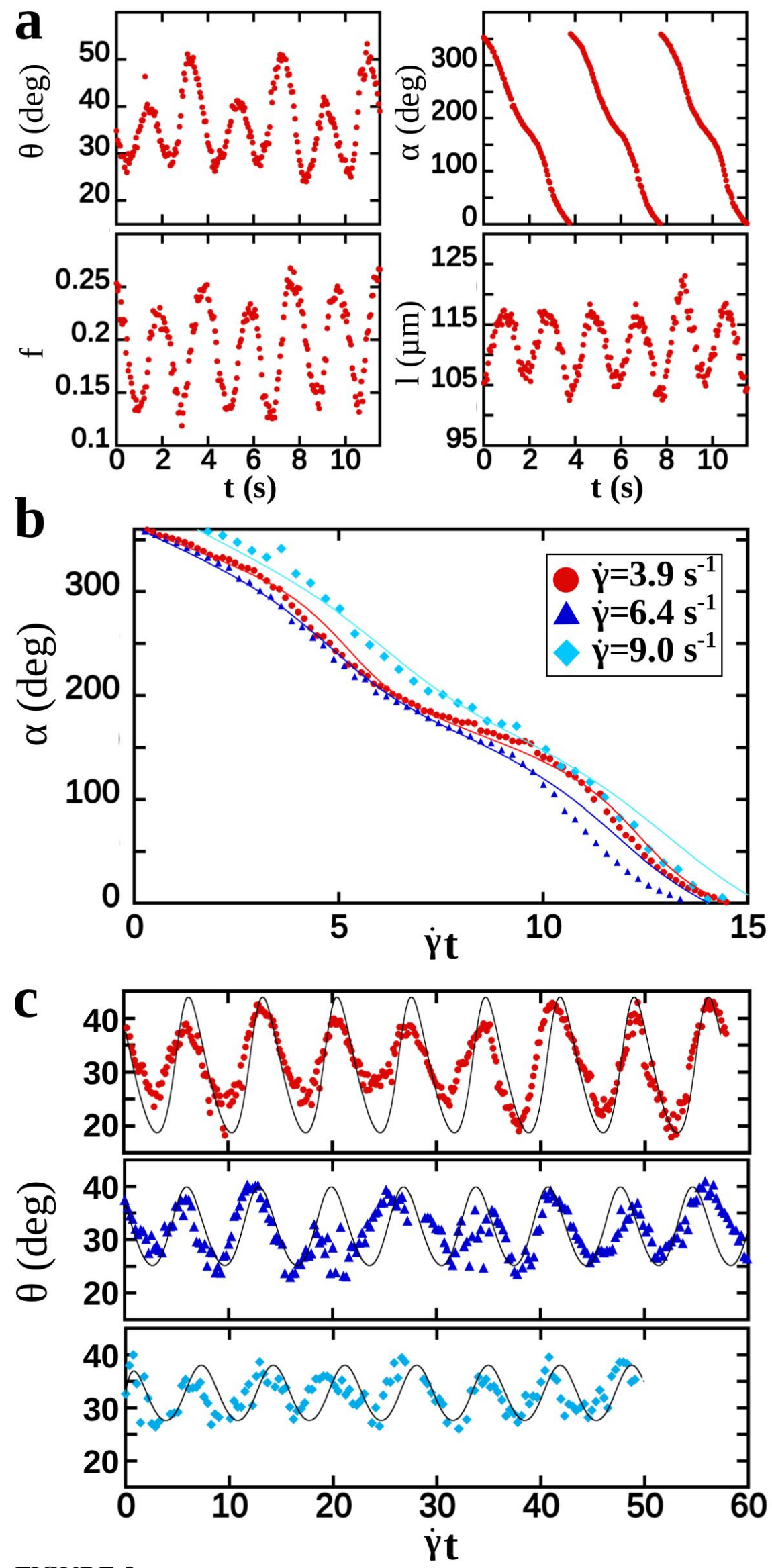

FIGURE 3 
a

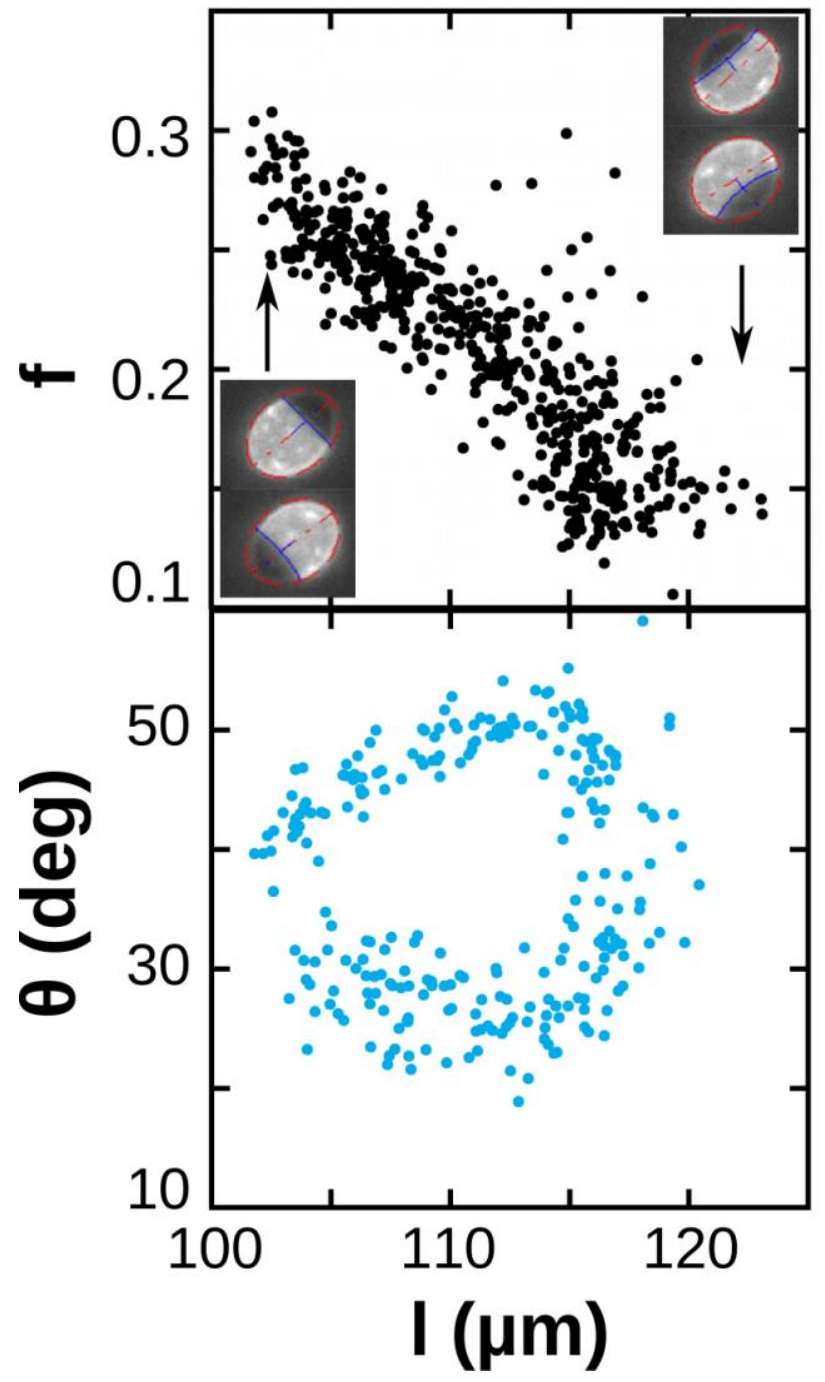

b

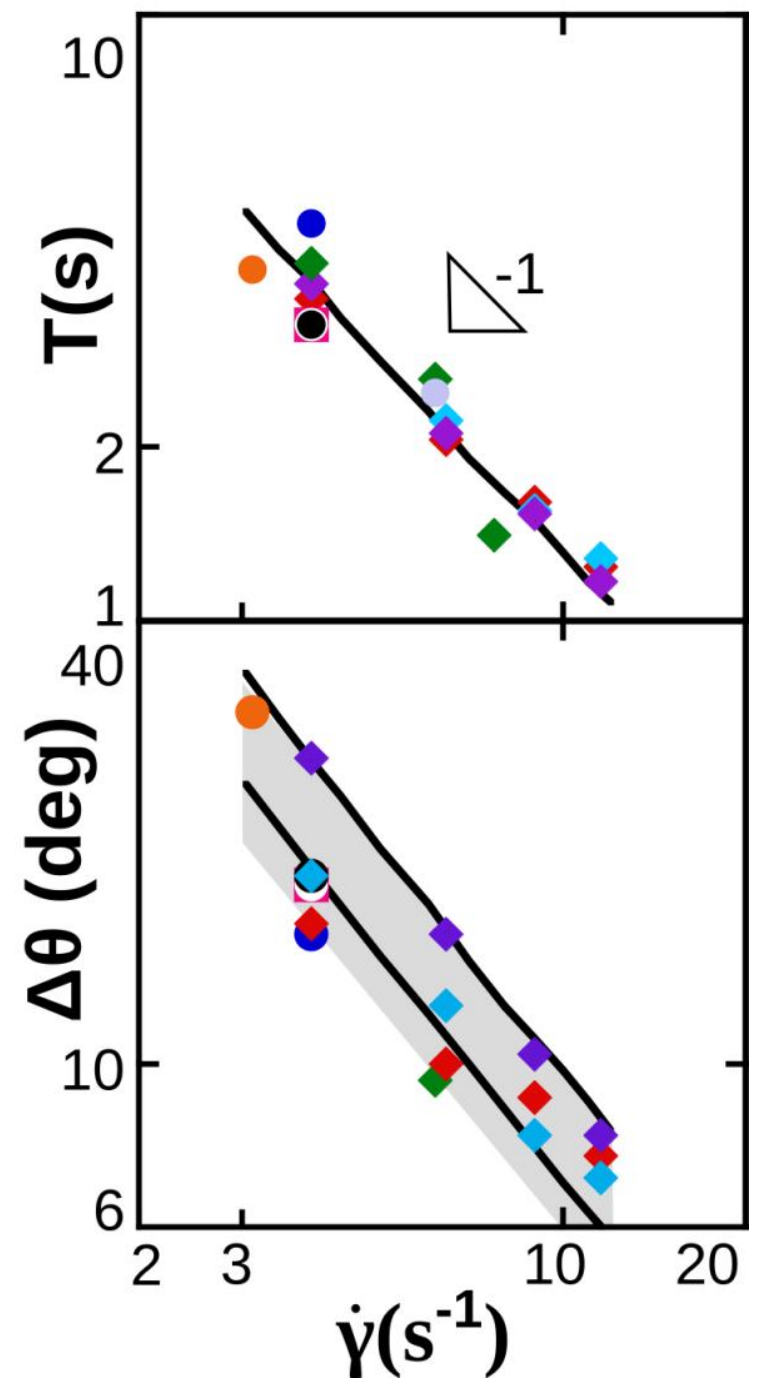

FIGURE 4 

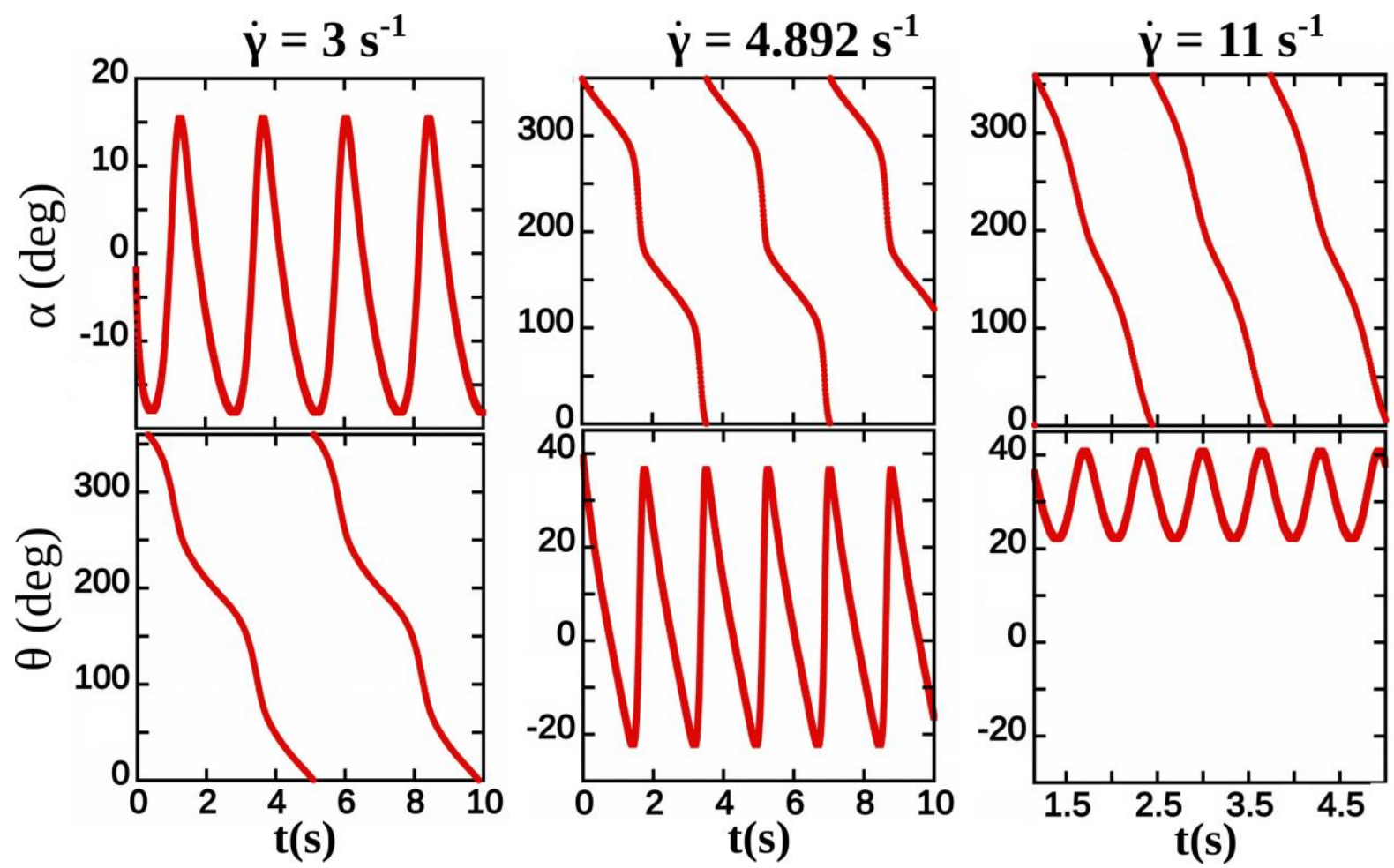

FIGURE 5
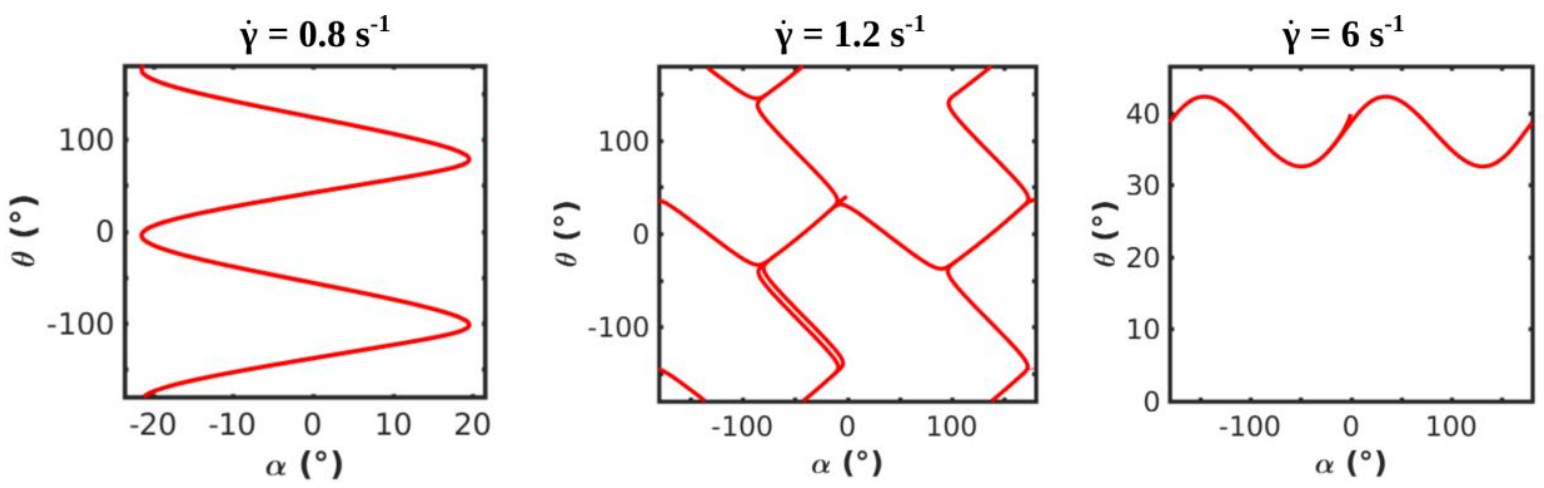

FIGURE 6 


\section{REFERENCES}

${ }^{1}$ M. Abkarian, C. Lartigue, and Annie Viallat, Tank Treading and Unbinding of Deformable Vesicles in Shear Flow: Determination of the Lift Force, Phys Rev Lett 88, 068103 (2002)

${ }^{2}$ P. M. Vlahovska, R. S. Gracia, Dynamics of a viscous vesicle in linear flows, Phys. Rev. E 75, 016313 (2007).

${ }^{3}$ A. Walter, H. Rehage, and H. Leonhard, Shear induced deformation of microcapsules: shape oscillations and membrane folding, Colloids Surf. A 183, 123 (2001).

${ }^{4}$ P. Erni, P. Fischer, and E. Windhab, Deformation of single emulsion drops covered with a viscoelastic adsorbed protein layer in simple shear flow, Appl. Phys. Lett. 87, 244104 (2005).

${ }^{5}$ M. Abkarian, M. Faivre, and A. Viallat, Swinging of Red Blood Cells under Shear Flow, Phys Rev Lett 98, 188302 (2007)

${ }^{6} \mathrm{H}$. Goldsmith and J. Marlow, Flow behavior of erythrocytes. I. Rotation and deformation in dilute suspensions, Proc. R. Soc. B 182, 351 (1972).

${ }^{7}$ T. M. Fischer and H. Schmid-Schonbein, Tank tread motion of red cell membranes in viscometric flow, Blood Cells 3, 351 (1977).

${ }^{8}$ T. Fischer, M. Stohr-Liesen, and H. Schmid-Schonbein, The red cell as a fluid droplet: tank tread-like motion of the human erythrocyte membrane in shear flow. Science 202, 894 (1978).

${ }^{9}$ J. Deschamps, V. Kantsler, V. Steinberg, Phase Diagram of Single Vesicle Dynamical States in Shear Flow, Phys. Rev. Lett., 102, 118105 (2009)

${ }^{10} \mathrm{~S}$. Keller and R. Skalak, Motion of a tank-treading ellipsoidal particle in a shear flow, J. Fluid. Mech. 120, 27 (1982).

${ }^{11}$ J. Dupire, M. Socol, and A. Viallat, Full dynamics of a RBC in shear flow, Proc Natl Acad Sci U S A 109 (51), 20808, (2015)

${ }^{12}$ D. Cordasco, A. Yazdani, and P. Bagchi, Comparison of erythrocyte dynamics in shear flow under different stress-free configurations, Phys. Fluids 26 (4), 041902 (2014)

${ }^{13}$ K. Sinha and M. D. Graham, Dynamics of a single red blood cell in simple shear flow, Phys. Rev. E 92, 042710 (2015)

${ }^{14}$ J. Dupire, M. Abkarian, and A. Viallat, Chaotic dynamics of red blood cells in a sinusoidal flow, Phys. Rev. Lett. 104, 168101(2010)

${ }^{15}$ T. M. Fischer, Shape memory of human red blood cells, Biophys. J. 86, 3304 (2004)

${ }^{16}$ J. Dupire, M. Abkarian, and A. Viallat, A simple model to understand the effect of membrane shear elasticity and stress-free shape on the motion of red blood cells in shear flow, Soft Matter 11, 8372 (2015)

${ }^{17}$ J. M. Skotheim and T. W. Secomb, Red Blood Cells and Other Nonspherical Capsules in Shear Flow: Oscillatory Dynamics and the Tank-Treading-to-Tumbling Transition, Phys.

Rev. Lett. 98, 078301 (2007)

${ }^{18}$ Z. Peng, A. Mashayekh and Q. Zhu, Erythrocyte responses in low-shear-rate flows: effects of non-biconcave stress-free state in the cytoskeleton, J. Fluid Mech. 742, 96 (2014)

${ }^{19}$ M. A. Mader, V. Vitkova, M. Abkarian, A. Viallat, and T. Podgorski, Dynamics of viscous vesicles in shear flow, Eur. Phys. J. E. 19, 389 (2006)

${ }^{20}$ M. Staykova, R. Lipowsky and R. Dimova, Membrane flow patterns in multicomponent giant vesicles induced by alternating electric fields, Soft Matter 4, 2168 (2008)

${ }^{21}$ See Supplemental Material [SuppNovember] for the calculation of the geometric parameters from the vesicle image. 
${ }^{22}$ see Supplemental Material [Movie_S1] for a movie of flowing GUV \#1

${ }^{23}$ see Supplemental Material [MovieS2] for a movie of flowing GUV \#1

${ }^{24}$ see Supplemental Material [Movie_S3c] for a movie of flowing GUV \#9

25 see Supplemental Material [Movie_S4] for a movie of tumbling GUV \#10

${ }^{26}$ see Supplemental Material [MovieS5] for a movie of flowing GUV \#3

${ }^{27}$ See Supplemental Material [SuppNovember] for the temporal evolution of the phase angle at increasing shear rate (Fig. S1)

${ }^{28}$ See Supplemental Material [SuppNovember] for a vesicle with arbitrary surface area ratio between the two lipid phases

${ }^{29}$ see Supplemental Material [MovieS6] for theoretical phase trajectories of $\theta$ versus $\alpha$ of a tumbling GUV

${ }^{30}$ see Supplemental Material [MovieS7] for theoretical phase trajectories of $\theta$ versus $\alpha$ of a GUV at the tumbling-tanktreading transition

${ }^{31}$ see Supplemental Material [MovieS8] for theoretical phase trajectories of $\theta$ versus $\alpha$ of a tanktreading GUV

${ }^{32}$ A. Tian, C. Johnson, W. Wang, and T. Baumgart, Line tension at fluid membrane domain boundaries measured by micropipette aspiration, Phys. Rev. Lett. 98, 208102 (2007)

${ }^{33} \mathrm{M}$. Trejo and M. Ben Amar, Effective line tension and contact angles between membrane domains in biphasic vesicles, Eur. Phys. J. E 34, 8 (2011)

${ }^{34}$ See Supplemental Material [SuppNovember] for both experimental and theoretical temporal evolution of $\theta$ and $\alpha$ for the tumbling GUV \#10

${ }^{35}$ T. Baumgart, S. T. Hess and W.W. Webb, Imaging coexisting fluid domains in biomembrane models coupling curvature and line tension, Nature, 425(6960), 821 (2003)

${ }^{36}$ J. S. Sohn, Y-H Tseng, S. Li, A. Voigt, and J. S. Lowengrub, Dynamics of multicomponent vesicles in a viscous fluid, J. Comp. Phys. 229 (1),119 (2010)

${ }^{37}$ K. Liu, G. R. Marple, J. Allard, S. Li, S. Veerapaneni and J.Lowengrub, Dynamics of a multicomponent vesicle in shear flow, Soft Matter 13, 3521 (2017)

${ }^{38}$ W. Helfrich, Elastic Properties of Lipid Bilayers: Theory and Possible Experiments, Z. Naturforsch. C 28, 693 (1973) 